\title{
Invasive Fungal Sinusitis: An Overview
}

\author{
Kailashnath Sant
}

Consultant, Sahyadri Speciality Hospital, and Sant Hospital, Pune, Maharashtra, India

Correspondence: Kailashnath Sant, Consultant, Sant Hospital, Survey No.1, Plot No. 117, Wadgaon Sheri, Pune-411014 Maharashtra, India

\begin{abstract}
Currently two main categories of fungal sinusitis are described; invasive and noninvasive. The invasive type can be; 1. Acute, fulminant, 2. Chronic, indolent, and 3. Granulomatous type. The noninvasive types are more common of which Allergic fungal sinusitis is commonly present in almost all extensive polyposis cases in India. The other noninvasive type is mycetoma or fungal ball, which carries the best prognosis if treated surgically well. The acute invasive fugal infection of sinuses by mucormycosis is an aggressive opportunistic infection caused by phycomycetes, first described in 1885 by Paltauf. The genera most commonly causing mucormycosis are Mucor or Rhizopus. Sinus and nasal infection by mucor causes orbitocerebral spread. Other organs involved are the lungs, GI tract and skin. Rhinorbito-cerebral mucormycosis has a mortality rate as high as $50 \%$, since they occur in immunocompromised state like uncontrolled diabetes, metabolic acidosis, chronic renal failure, AIDS, leukemia, etc.
\end{abstract}

Keywords: Fungal sinusitis noninvasive.

\section{INTRODUCTION}

Chronic rhinosinusitis is a common affliction of the nose and paranasal sinuses, of which, the fungal infections are commonly seen in India. These are seen in both immunocompromised as well as immunocompetent patients. Our experience in this subject, in the last 15 years supports this fact.

Almost all cases of extensive bilateral sinonasal polyposis are associated with allergic fungal sinusitis.

\section{CLASSIFICATION}

\section{Fungal Sinusitis}

\begin{tabular}{lcccc}
\hline \multicolumn{2}{c}{ Noninvasive fungal sinusitis } & \multicolumn{3}{c}{ Invasive fungal sinusitis } \\
\hline \multirow{2}{*}{ NIFS } & IFS \\
NIFS-A & NIFS-B & IFS-A & IFS-C & IFS-G \\
AFS & Fungal ball & Acute & Chronic & Granuloma \\
Allergic FS & Mycitoma & \multicolumn{3}{c}{ Invasive } \\
\hline
\end{tabular}

The mainstay of treatment is surgical. With the advent of sinus endoscopes, it is today possible to surgically treat almost all such fungal infections radically, yet minimally invasively. The use of antifungal agents is mandatory in the invasive variety, although some noninvasive infections may also require postoperative medical therapy. Overall the prognosis of NIFS is good, although relapses are common in AFS. Mycetomas (fungal ball) have the best results, since surgical clearance is usually curative. All invasive forms ultimately have a poor prognosis; in fact, the mortality rate is more than $50 \%$, which is compounded by the underlying immunocompromise.

\section{COMMON PATHOGENS}

\section{NIFSA-Allergic FS}

1. Aspergillus Fumigatus.

2. Curvularia Lunata.

3. A Bipolaris.

4. A Drechslera.

\section{NIFSB_Fungal Ball, Mycetoma}

1. A Fumigatus.

2. Dematicious Fungi.

\section{IFSA —Acute Invasive Fulminant FS}

1. Saprophytic Mucorales-Mucormycosis.

2. Rhizopus, Rhizomucor.

3. Absidia Mucor.

4. Cunninghamella.

5. Mortierella.

6. Saksenaea.

7. Apophysomyses Species.

\section{IFSC—Chronic Invasive FS}

Aspergillus Fumigatus. 


\section{IFSG -Granulamatous}

Aspergillus Flavus.

\section{REVIEW OF LITERATURE}

Blitzer et al, studied 179 cases of acute invasive FS having $70 \%$ prevalence of diabetes, $26 \%$ had other underlying diseases such as leukemia, renal disease, infant diarrhea, post-transplant immunosuppression, pancreatitis. Only 4\% had no identifiable risk factor.

Blitzer and Lawson's study suggests the following common clinical signs of mucormycosis:

- Cranial nerve deficit

- Proptosis

- Facial swelling

- Palatal ulcer

- Stupor and coma in the order of severity.

Blitzer and Ochi concluded that acute IFS needs surgery and systemic antifungal agents. With radical debridement, the survival rate was $76 \%$ while the rate dropped to $57.5 \%$ with medical treatment alone. They could achieve $81 \%$ survival rate by combining surgery and Amphotericin B therapy. The underlying cause of the immunocompromised state was taken care of adequately in all cases.

\section{PATHOPHYSIOLOGY AND CLINICAL PRESENTATION OF ACUTE INVASIVE FUNGAL SINUSITIS-MUCORMYCOSIS}

The spores of these fungi are ubiquitous and gain entrance to the human body through the mouth and the nose. ${ }^{1}$ Immunocompetent individuals mount a macrophage response, which will phagocytose these spores; thus they do not develop the disease. ${ }^{2,3}$ Immunocompromised patients are unable to mount such a response, allowing germination of spores to form hyphae, which eventually result in vascular invasion.

1. Rapid spread through vascular invasion into orbit and CNS.

2. Toxic, severely ill-patient, febrile with cough, nose discharge and headaches.

3. Changes in mental status.

4. Needs urgent hospitalization.

5. Immediate surgical intervention and systemic antifungal drugs.

6. Aggressive control of basic cause like diabetes, renal function.

\section{CHRONIC INVASIVE FUNGAL SINUSITIS}

1. Progressive fungal infection.

2. Diabetics with fluctuating sugar levels.

3. Low grade invasiveness.

4. Classical features of orbital apex syndrome.

Due to mass in the superior part of orbit causing diminished vision and ocular immobility.

\section{GRANULOMATOUS INVASIVE FUNGAL SINUSITIS}

Exclusive to immunocompromised patients of North Africa - proptosis!

\section{IMAGING STUDIES}

CT Scan and MRI Scan:

- Both have to be in coronal and axial cuts with and without contrast.

- Study the opacity for variable density-shadows of Ca and Mg crystals (AFS).

- Look for the confines of sinuses - expanding boundaries towards orbit and brain (remodeling of sinus boundaries).

- Note if destruction of bony trabeculae, or bony boundaries.

- Differentiate mass from secretions to know the real extent of disease.

- See for orbit and brain involvement.

\section{MEDICAL TREATMENT}

1. Systemic steroids

- Given postsurgery, after suspecting fungal infection or confirming it

- Preferably low dose therapy

- $0.5 \mathrm{mg} / \mathrm{kg}$ prednisolone alternate day in tapering dose for 3 months with all routine monitoring.

2. Topical nasal steroid spray.

3. Salt water nasal douches.

4. Systemic antifungal agents

- Only in invasive FS

- Pre- and postoperative - Amphotericin B

- Ketaconazole

- Itraconazole

- Posaconazole.

5. Antibiotics and antianerobics with mucolytics.

\section{PERSONAL EXPERIENCE}

For all types, the treatment of choice is surgery, under general anesthesia. 


\section{Preferably}

Endoscope assisted total sphenoethmoidectomy with frontal recess exploration.

- Explore all sinuses

- Widely open all ostia for good postoperative look and drainage

- Fascilitating easy douching

- Correct septal deviations and spurs, although minor, to create good nasal space bilaterally.

- Bulky or polypoidal middle turbinate should be appropriately shaved or cut; usually mucor patients will not bleed until normal mucosa free from disease is reached, however microscopic spread may cause necrosis in postoperative period, which warrants repeated surgical interventions and debridement under cover of antifungal drugs. In spite of good surgery, relapses are common, with the exception of mycetomas, which usually do not recur. Postsurgical facial disfigurement is an issue, when the palate, alveolar margin or orbit are sacrificed, and will need reconstruction and rehabilitation, if the patient survives. In some cases, an external approach can be considered in cases of Frontal sinus involvement. In old patients, the prognosis of mucormycosis is very poor. The role of local irrigation of the sinus cavity and orbit, and drug delivery through packs soaked in Amphotericin $\mathrm{B}$ improves results. The medication does not reach necrotic and poorly perfused tissues due to poor vascular supply. This in effect results in further disfiguring surgery due to repeated debridements. Recently we have started hyperbaric oxygen therapy, however, our experience of this therapeutic modality is limited.

\section{DISCUSSION}

In our experience, patients with bilateral extensive polyposis invariably show thick mucus lodged in the sinus cavities. These test positive for fungus in the mucus but, negative in mucosal biopsy and are hence termed 'noninvasive'. The aggressive surgical clearance of this mucus at the time of surgery, along with postoperative systemic steroids and topical steroid sprays reduces the rate of recurrence. All the diseased mucosa needs to be addressed and the end point of endoscopic clearance of disease should demonstrate the maxillary, sphenoidal and frontal ostia to be widely exposed to the empty ethmoid cavity, with the middle turbinate medially, skull base postero-superiorly and the lamina papyracea laterally. If required one should not hesitate to correct a septal deviation to enable good visibility and easy access to all areas. The same surgical protocol is to be followed for fungal ball/mycetoma. Surgery for invasive fungal sinusitis (mucormycosis) is radical, and the end stage of disease is until bleeding occurs from the dissected area indicating normal tissue has been reached. However, repeated debridements are done as and when required depending on the patient's clinical improvement, in combination with Amphotericin B. To improve the outcome of the therapy in invasive fungal rhinosinusitis, we follow a comprehensive approach involving the endoscopist (ENT surgeon), ophthalmologist, diabetologist, neurophysician, intensivist, and oculoplastic surgeon with a neurosurgeon; all treating the same patient in the different stages of the disease.

\section{CONCLUSION}

A comprehensive outlook towards the surgical and medical management in a case of invasive fungal disease results in an improvement in the survival rate. ${ }^{4}$

\section{REFERENCES}

1. Blitzer A, Lawson W, Meyer BR, Biller JF. Patient survival factors in paranasal sinus mucormycosis. Laryngoscope 1980;90:635-48.

2. Blitzer A, Lawson W. Mycotic infections of the nose and paranasal sinuses. In: English G, Otolaryngology. St. Louis, Mo: JB Lippincott 1992;1-23.

3. Blitzer A, Lawson W. Fungal infections of the nose and paranasal sinuses. In: Otolaryngology Clinics of North America: Fungal Infections of the Head and Neck 1993;26:1007-35.

4. Ochi JW, Harris JP, Feldman JI, Press GAA. Rhinocerebral mucormycosis: Results of aggressive surgical debridement and Amphotericin B. Laryngoscope 1988;98:1339. 